\title{
The effect of dynamic social material conditions on cognition in the biomedical research laboratory
}

\author{
Chris Goldsworthy ${ }^{1}$ (D)
}

Published online: 1 November 2018

(C) The Author(s) 2018

\begin{abstract}
The modern biomedical research laboratory is increasingly defined by dynamic social material conditions requiring researchers to traverse multiple shifting cognitive ecologies within day-to-day practice. Although the complexity of biomedical research is well known, the mechanisms by which the social and material organisation of this space is negotiated has yet to be fully considered. Integrating insights from Material Engagement Theory and Enactive Cognition with observations undertaken within a biomedical research laboratory, this paper develops an understanding of how actors are able to respond to the dynamically changing world that is enacted with and around them.
\end{abstract}

Keywords Material engagement theory $\cdot$ Enactive cognition $\cdot$ Metaplasticity

\section{Introduction}

Modern biomedical research is increasingly defined by overlapping social and material arrangements (Keating and Cambrosio 2003) to the extent that biomedical research practice cannot be effectively carried out in isolation of the surrounding social institutions. As such, biomedical research scientists are required to negotiate multiple cognitive worlds within their day-to-day practices; however, the ways in which this complexity is negotiated within situated laboratory practice has yet to be fully examined. Drawing on Material Engagement Theory (MET) (Malafouris 2013) and Enactive Cognition (Valera et al. 1991; Di Paolo 2009; Stewart et al. 2011; Hutto and Myin 2013; Gallagher 2017), this paper will examine how research scientists respond to, and are configured by, the shifting complexes of socio-material relations within biomedical

Chris Goldsworthy

Christopher.goldsworthy@insis.ox.ac.uk

1 Institute for Science, Innovation and Society, University of Oxford, 64 Banbury Road,

Oxford OX2 6PN, UK 
research practice. More specifically, I will empirically explore the three working hypotheses of MET through three situated case studies;

- Enactive signification and the safety goggles as a material sign;

- Material agency and the production of the Western Blot;

- The extended mind and the place of the lab book in (re)producing experimental protocols.

These case studies are examined as a way of shedding light on the dynamic organisation and re-organisation of practice in this space and its impact in the forms of cognition enacted by the research scientists. In exploring this, the three hypotheses of MET are configured as central mechanisms by which actors shift across institutional conditions within situated biomedical research practice.

\section{Laboratory lives}

The socio-material and spatial organisation of the laboratory space has long been positioned as the locus of scientific knowledge (re)production (Latour and Woolgar 1979). Lynch (1991) questions this singular institutional understanding and develops the concept of Topical Contexture as situation bound complexes of human and technological actions, which serves to transform the 'place' of scientific knowledge production. This contribution transformed the laboratory from an institutionally static, bounded set of actors and technologies, to positioning scientific production as a consequence of human - technological complexes which may extend far beyond the laboratory. Much as Annemarie Mol (2002) observed in relation to Atherosclerosis, it would be a great reduction to view the biomedical research laboratory as a singular ontological entity.

Biomedicine in particular is described as a cobbled together socio-technical system of standards, practices and infrastructures serving to produce knowledge, and diagnose and treat patients (Lock and Nguyen 2018). Drawing on MET and Enactive Cognition, I argue that the social and material organisation of particular institutional frames of practice configure the researchers in the biomedical research laboratory within changing cognitive worlds that constitute modern biomedical research. Moreover, the practical negotiation of this complex institutional ecology is positioned as an essential facet of the organisation of biomedical research and the exploration of this will constitute the empirical focus of this paper.

\section{Methods and materials: observing cognitive flexibility}

The situated case studies are drawn from observations across the complex organisation of research in the field of inherited cardiac conditions (ICC) and cardiac genetics/ genomics within a biomedical research laboratory, as well as qualitative interviews with key professionals from within this space. The research observed in this laboratory was designed to unpick the functional, disease causing mechanisms of genetic variants associated with ICC's linked to sudden death at a young age. The observations were carried out over a 6-month period between 2017 and 2018. These observations 
focussed on the every-day practices of the researchers and their negotiation of the material world around them across a variety of practices, including; sample preparation, experimental practice, data analysis and experimental trouble shooting. The focus of these observations was upon the organisation of social and material relations within and between significant periods of situated practice, or across specified bounded tasks. The researchers themselves defined the delineation of tasks.

\subsection{The biomedical research laboratory}

The social and spatial organisation of this laboratory serves to emphasise the complex entanglement the research ongoing within this laboratory, has with clinical services. The laboratory is located in the research wing of a large hospital in the UK. It is no accident that the laboratory is in such close proximity to the clinic spaces; such a coresidence facilitates the physical mobility of actors and resources (tissue samples and patient information) between sites as well as facilitating a mutual awareness of the practices ongoing in both spaces. The principle investigator of the research projects that I was able to observe is also primarily a clinical cardiologist. The laboratory research relies upon this relationship as many local clinical patients constitute the bodily samples that are the focus of research investigations, and reconstructions of the very specific genetic variants found in these patients often constitute the objects of experimentation. While this paper will only report on observations undertaken within the laboratory, the proximity of the clinic is ever-present and as such, the cognitive negotiation of the clinical world significantly shapes the practices in and around the laboratory.

The laboratory is organised into wet and dry areas, this is increasingly indicative of biomedical research following the increase in prominence of bioinformatics, and the co-dependence of computational and physical modes of investigation within everyday life sciences laboratory practice (Bartlett et al. 2017). Computers line the three outer walls of the laboratory, with each researcher having their own desk space. The central space in the laboratory is used for the wet lab work, that is, the experimentation undertaken on biological material or chemicals that may be hazardous. Within this lab, there are many different research groups all conducting biomedical research associated with cardiac or circulatory conditions. Much in the same way discussed by Keating and Cambrosio (2003) the disciplinary constitution of the researchers within this laboratory is heterogeneous; with research scientists trained in clinical chemistry, molecular biology and genetic sciences. When asked about how this affects the research undertaken, the importance of disciplinary identity was downplayed; emphasis was instead put on the practical abilities of the researchers. Indeed the experience of researchers in particular novel techniques, such as Induced Pluripotent Stem Cell (IPSC) culturing, are positioned as some of the key distinguishing features between researchers in practice. Identity within experimental practices within this laboratory is intimately associated with the researcher's proficiency in engaging with the materials and technologies of the laboratory in meaningful way, this is perhaps a consequence of the career stage of the laboratory workforce. Whilst the official distribution of those employed to work in the laboratory is stratified from clinical professor to $\mathrm{PhD}$ student, in practice the day-to-day workforce was primarily represented by post-doctoral researchers, those at lecturer or professor grade rarely visited the laboratory despite being the named principle investigators on all of the ongoing research projects. 
The research project examined on within this paper focussed on understanding specific functional consequences of genetic variants upon the expression of an ICC. The research specifically investigated variation in a large complex gene and the consequences of this variation upon the structure and function of the protein it encodes. At the time of the observations, this research was reaching its formal completion and many of the practices I have been able to observe have been working towards the production of a final publication. Before empirically exploring the embodied relational practices of this research through lens of MET, I will briefly outline the theoretical position taken within regards to the enactment of plural cognitive institutions within, and extending beyond the laboratory infrastructure.

\section{Shifting worlds, changing minds}

Enactive Cognition holds that the brain is not representing the world, but is responding to it (Gallagher 2017); such an assumption positions cognition as a relational process (Maturana and Varela 1980; Di Paolo 2009; Thompson and Stapleton 2009; Newen et al. 2018). Positioning the brain as relationally entangled with the body and the material environment (Gosden 2008; Malafouris 2004, 2008), 'Being-in-the-world' (Heidegger 1927, p. 84), is integral to Enactive (Valera et al. 1991; Di Paolo 2009), Extended (Clark and Chalmers 1998) and Distributed theories of cognition (Hutchins 1995a, b; Hollan et al. 2000). These approaches liberate the mind from within the head of individual actors. However, as noted by Merleau-Ponty (1962), the body at once enables and restricts perception to that which is afforded (Gibson 1977) by its senses. Whilst there are specific material and social adaptations that can facilitate or restrict the engagement the body can have with the world; from the relationship between the blind man and his stick, through to the cardiac implantable defibrillator and the patient (Oudshoorn 2015), there is a sense in which this is restrictive. However, the organisation of the socio-material world that we enact, that we engage with as we go about our daily lives, is dynamic and flexible. If we are to accept that human agency and cognition cannot be disentangled from the social and material environment which they situationally enact, it stands to reason that the mind of the researcher will change as they traverses the multiple socio-material conditions of the laboratory in situated practice.

Existing examinations of forms of cognition that entangle the brain, body and environment (material and socio-cultural) tend to focus on institutionally static examples; the man with impaired memory function and his notebook (Clark and Chalmers 1998), the pilot and his/her cockpit (Hutchins 1995a, b), the potter and his/her clay (Malafouris 2013). But the pilot is not always, and never only, flying the airplane, the potter is not always working with clay and the man with an impaired memory function does not have to rely on his notebook to perform every function. Travelling across socio-material institutional conditions is a defining characteristic of the everyday lives of humans, yet the cognitive shifts that occur as a consequence of this have yet to be fully explored.

\subsection{Dynamic material engagement across institutional ecologies}

Cognitive institutions constitute our relational and emergent metacognitive architecture. By this, I mean to say that cognitive institutions are not pre-ordered arrangements of 
things in time and space, whose relations exist outside of the disciplined enacted situation. They are crucially dependent upon the dynamic relations between humans, materials, space and culture. No person can enter the laboratory and immediately become fully entangled within the socio-material relations unfolding in practice. Institutional agency is not guaranteed by simply being physically located in a space amongst the people and things, this results in a "bodied individual in a physical space" (Adam 1998, p. 136), the mind of the researcher in this scenario is incomplete (Malafouris 2015; 2016a, b)), in terms of its orientation to the institutional organisation of the laboratory. Disciplined participation is necessary for cognitive assimilation. Gallagher (2013) positions institutions as integral in shaping our cognitive processes, defining the institution as an emergent, dynamic process from which we inherit the cognitive processes of our ancestors or predecessors (human and material, See Goodwin 2017). Gallagher goes on to say that it is our engagement with these institutions, in participation with others that further shapes our collective cognition.

Malafouris (2013) concisely identifies the mechanisms by which the human mind is co-constituted within the entanglement of the brain, body and material culture, or the socio-material organisation of institutions. His discussion of Metaplasticity (Malafouris $2009,2010 ; 2013 ; 2015)$, hinges on the assumption that we have plastic minds embedded in the unfolding of plastic material culture. This evolutionary epistemology (Malafouris 2013; Iliopoulos 2016), in which variation of the mind and social behaviour is tightly intertwined with ones embeddedness within the processes of situated material culture, is helpful in that it positions the locus of cognition not in either the human or material actor, nor the collectivity of social and material arrangements, but in the relations between them. Where Malafouris examines the semiogenic evolution of the mind and material culture, from an archaeological perspective, over a protracted period of time. In this paper, I take insight from his understanding of the co-constitutive entanglement of the brain, body and material culture in the production and reproduction of ways of thinking at the micro temporal scale of the biomedical research laboratory. I argue that mutually constituted forms of cognition are made and remade through the engagement of people and things, as the researcher negotiates the institutional complexities of the laboratory. In exploring this, I will draw explicitly on the three working hypotheses of MET; the extended mind, enactive signification, and material agency (Malafouris 2010, 2013). Each of these hypotheses will be explored via empirical case studies of laboratory practices.

\section{Active material engagement: enactive significations}

There is no sense in which Material Engagement is idle; indeed relational participation is essential to this process (Malafouris 2013). Yet relational activity deserves further emphasis as a way of developing an understanding of shifts in thought, as facilitated through active engagement with the material and institutional surrounding. It makes little sense to talk about the material surrounding or equipment in the laboratory as tools or props in a dramaturgical sense (Goffman 1956). The microscope is not used to perform the role of researcher, nor does the microscope represent scientific culture; but in relational engagement with the researcher, the microscope and the environment serve to reproduce a practical experimental way of thinking. MET posits that materials or 
technologies engaged with within situated practice enact the world around them in the same way, explicating the way of thinking to those privy to the institutional pedagogy. Contemporary discussions of the concept of Enactive Signification (Malafouris 2013; Iliopoulos 2016) are helpful in exploring this.

Malafouris (2013) defines Enactive Signification as "the process of "conceptual integration" responsible for the co-substantial symbiosis and simultaneous emergence of the signifier and signified" (99). The significance of this definition is the assumption of the institutionally embedded co-constitution of the material sign. The material does not enact any world when disentangled from the situated relations in which the enactment is taking place. In making this claim Enactive Signification overcomes the problem of representation inherent in disembodied approaches to material semiotics. Material objects do not act as vessels for a particular way of thinking, the signification is emergent within situated action; although this is not to say that a material or technology does not mediate the potential actions based on the limitations of its physical structure, the technology has limited affordances (Gibson 1977).

A helpful way of exploring this is through the plurality of signification of the fairly benign laboratory technology, safety goggles, as they are engaged with in practice. Here I will examine the multiple 'enactive effects' of the goggles as a material sign (Malafouris 2013). The goggles are not used in the lab as a way to signify identity as a researcher, nor are they simply a safety precaution; they are instead engaged with on multiple levels and are considered to be part of the structural power relations in the laboratory. The researcher selectively wears goggles, on the basis that unless they are doing something dangerous, the goggles actually hinder much of the necessary precise observations and practices. For example, when pipetting microliters of valuable patient sample into an agarose gel, the researcher will not wear goggles, as within this situated practice they do not support the production of the world of the experimental researcher. This way of thinking is in direct conflict with the intractable safety dynamic of the laboratory environment. In this laboratory, goggles are mandatory when at the lab bench; this is formally inscribed into the standards of practice. The choice not to wear the goggles requires the conscious negotiation of forms of social cognitive practice based upon the plurality of relations the goggles afford. The goggles at the same time signify the power relations between the researcher and the governance of local laboratory practices, the ability of the goggles to protect eyes from chemical splashes, and the constraining properties of the goggles on the ability of the researcher to be able to make precise observations. The goggles are at once, three distinct epistemic entities as the relational action unfolds at the intersection of three tractable institutional arrangements. It is only when the surrounding unfolding of action is taken into consideration, in terms of the ongoing experimental process, that sense can be made of the particular signification the goggles are enacting.

\subsection{The negotiation of plural conceptual blends in practice}

In the above section I position the safety goggles as a material anchor (Hutchins 2005) within multiple conceptual blends (Fauconnier and Turner 2002). Blended conceptual spaces emerges as a consequence of the coalescence of projections from two (or more) input spaces (Fauconnier and Turner 2002). A consequence of this is constructed as an emergent logic that does not equal the sum of the projected inputs. Hutchins (2005) has 
subsequently developed this to include the projection of material anchors into the blend, which serves to stabilize the emergent relations in situated practice.

The safety goggles, as a material anchor within the conceptual framework of safe laboratory practices is straightforward to configure. Within wet lab practices there exists the conceptual structure to protect one's self and others from harm. The wet lab is configured as a place in which hazards exist; this is embedded within the visible material structures of the laboratory designed to mediate risk; such as fire extinguishers and safety posters. In addition to this, the local oral history of the consequences of risky laboratory practices is performed when the issue of safety precautions arise. When discussing the use of goggles with the laboratory researcher, a story was told concerning a researcher who managed to get corrosive chemicals in their eyes on two separate occasions, despite following all safety procedures. It was reported that on the second occasion, the researcher accidentally splashed a small amount of hazardous liquid in their eye whilst pipetting, despite wearing goggles and working under a flow hood, in which there is a physical glass barrier between the researcher and the experimental process. Thinking about safety in the lab is not metaphoric or disembodied from local practices; it is tangible and is stabilized by institutional, situated material practices, embedded in the collective memory. Yet as discussed, this heightened conception of risk does not itself constitute a risky situation, after all, there is little conceived sense of risk when analysing and interpreting data at the computer, despite being only 1-2 m away from wet lab experiments. It is the integrated conceptual blend of the projection of personal risk with that of wet lab experimental practices that constitutes the emergence of the blended space. While the goggles could be positioned as part of the input space of risk, they are most closely aligned with experimental practices, as they are not enacted when not situated within experimental practice. And while both conceptual projections discussed involve mental and material processes, the goggles are positioned as a material anchor as they stabilize the emergent relational dynamics of safe experimental practice. Yet as discussed, goggles are not always worn in the lab.

Malafouris (2013) offers an extension to the examinations of conceptual integration by Foucannier and Turner (2002) and Hutchins (2005). He positions his interest in dynamic networks of integrative projections. While Malafouris (2013) does not explicitly explore plural potential of material anchors in practice, he positions conceptual blends within the material world in which meaning can be made and remade via embodied engagement. Thought and reasoning are not abstracted from the physical world furnished with the social and material processes that constitute it. It is these dynamic networks of embodied engagement with material anchors that offers the potential to consider the plurality of material signification in practice. Depending on the integrative network in which the goggles, as a material anchor, are embedded, the way in which they are engaged with by the researcher will change. Such a change in situated socio-material relations can be unpicked within a short frame of practice in the laboratory.

When preparing a large number of samples for a Polymerase Chain Reaction (PCR) based cloning protocol, the researcher will rarely wear protective goggles; the personal risk is low and the necessity for precision is high. The PCR preparation process begins with the creation of a mixture containing precise amounts of test DNA, DNA polymerase enzyme, primers, and nucleotides; the quantity of each is as little as a single 
microliter. When working with such small quantities, using fine pipette tips, it is essential that the researcher can see the tip fully submerge in the clear liquids and that no air bubbles remain in the tip, as this would affect the ratio in the mixture. The goggles in this case, obscure the view of the researcher, which could jeopardise the success of the reaction. The goggles signify impeded perceptual ability. When observing this preparation process on one occasion, the laboratory manager entered the lab, at the same time the researcher subtly shifted the goggles off the top of their head down on to their face. Although the laboratory manager did not interrupt the intricate process being undertaken, their presence in the lab shifted the relational organisation of practices. The embodied engagement between the researcher, the goggles and the materials involved in preparing the PCR was reorganised, the primary material sign enacted by the goggles was no longer that of the dynamic between perceptive ability and successful precision experimental practice, they instead signified the organisation of power relations in the laboratory. This shift occurred as a consequence of the dynamic shift in the constitution of the network of integrative projections. The addition of the laboratory manager, despite their lack of intervention, re-made the signification of the goggles within the dynamic embodied engagement amongst the materials of the laboratory.

In this section I have empirically examined the consequence of considering the material sign as enmeshed in a 'web of mutual dependence' (Hutchins 2010, 705) within, and across cognitive ecologies. The goggles enact an integrated projection of the network of relations in which they are situationally embedded. However, to be able to take seriously the consequence of enactive signification, as I have positioned it; that the goggles act as the material sign, from which cognitive relations shift, the goggles must be considered active and re-active across the relational cognitive ecology of the laboratory. This next section will explore how agency could be conceived of across the dynamic socio-material conditions of the biomedical laboratory.

\section{Material agency: western blot}

In keeping with Malafouris' (2013; Knappett and Malafouris 2008) definition, agency will be explored, not as an attribute of the material entity but as an emergent consequence of institutionally embedded situational practice. In exploring: "When and how agency is constituted" (147) Malafouris (2013) is offering the possibility that enactments of agency (human and material) vary significantly when relational conditions change. In this section, I will explore how material agency is constituted in the entanglement of situated practice in the laboratory within the conduct of one of the most common experimental practices observed, the production of the Western Blot. By decoupling agency from the actor in this way, I will also explore how the situated enactment of material agency shifts with the web of mutually entangled relations within the laboratory.

Western Blots are designed to identify specific proteins in a sample based upon molecular weight. The expression of these proteins is visually represented via the production of bands of different concentrations at different locations on a small, thin square $(8 \mathrm{~cm} \times 8 \mathrm{~cm} \times 1 \mathrm{~mm})$ of sodium dodecyl sulfate-polyacrylamide gel (SDS PAGE). The Western Blot is the product of the transfer of the marks on this gel onto a 
blotting membrane. Whilst there are quantitative tools designed to measure the position and concentration of each band produced on the Western Blot, the researchers tend to analyse the imaged blot by eye. In most cases, the researcher could see that one band was larger and darker than others, and situated adjacent to the appropriate molecular weight marker, signifying the concentration and presence of the protein concerned. However, the meaning and value attributed to this visual material would be difficult to consider outside of the socio-technical context in which it was produced.

The practice of producing and analysing a Western Blot is not a discrete event within a research project, there is often a period of weeks of preparation and experimentation which effects the order that the samples are loaded into the gel, the cleaning process, as well as the implicit expected outcome. These existing socio-material relations emerge in practice at the point of deciding which methodology to employ, whether this is the commercial lab kit (Thermo Fisher iBlot), or the manual production of the experimental protocol. There are practical considerations that inform which technique to employ; the commercial kit is certainly much quicker to run, but what is gained in efficiency is lost in experimental control over parameters. The commercial kits contain a detailed protocol, precast gels, all of the necessary buffers, reagents and consumables (Nitrocellulose stacks), as well as the proprietary dry transfer device. The manual approach requires researchers to cast their own gel, which can be done to their own specification, mix their own buffers, and conduct their own transfer, which can also be optimised in terms of time taken and the voltage of the electrophoresis.

The Western Blot protocol explored here employed the manual methodology, however over the course of the observations, tensions emerge which re-align the relational engagement between human and material agents in ways that drastically reshape the organisation of this practice.

Over a three-week period of observations, one researcher conducted, optimised and repeating the same Western Blot protocol. The function of this Western Blot was not to confirm the presence of a particular protein; it was to produce a clear image for publication. The researcher had previously successfully produced a Western Blot that identified the protein of interest but the transferred image contained residual artefacts. This outcome was anticipated, the information regarding the presence or absence of the protein was there and the aesthetics were not of particular concern at this point. Based upon this earlier experience the researcher chose to follow the same protocol, only changing the parameters thought to impact the clarity of the image produced. The gel was cast using a $12 \%$ polyacrylamide solution; the preference in the laboratory in general is a slightly higher percentage, which is thought to prevent leakage from the cast. This researcher used $12 \%$ as this enables the proteins to run better, which is preferential when producing an image for publication, but makes double the amount to account for the inevitable leakage.

When planning the loading of the gel with the sample solution, the researcher weighs up the intended function of the gel (to produce a clear image) with the situated previous experience. The previous experimentally successful Western Blot used a very small amount of sample; during the situated optimisation process, the researcher positions this as a potential reason for the artefacts in the first Western Blot. There was also the concern as to whether there was enough of the experimental sample remaining to multiply the amount used. These economic concerns and embodied experiential socio-material relations impact the unfolding of action in significant ways. If the remaining patient sample was too small, less would be loaded into the gel, as it 
would be very difficult to source more; if the previous Western Blot provided a clearer image, or a different cause of the artefacts in the image were considered, perhaps the experiment would not be repeated, or a different material parameter would be changed. Within these mutually entangled relations, the researcher maintains the ability to influence the situated relations as they unfold, as mediated by the affordances of the materials engaged with. As a consequence of this dynamic web of practice, in which there is more than enough experimental sample left, and based upon the previous experimental findings, 3 times the amount of sample is loaded, which is restricted only by the physical structure of the wells in the gel that can only hold $15 \mu$. This decision was only made possible by the relational enactment of the researcher and the materials as the production of the Western Blot, as embedded within the broader research project, unfolded over time.

Following the transfer of the gel, after each optimisation of the Western Blot protocol, the researcher would carefully compare the location and concentration of the bands in the existing, experimentally successful but not publication worthy blot, with the new outputs, which were beautifully clean, but did not convey the data. After multiple successive attempts, using both the pre-made kit and the manual method described, a problem was identified with the antibody which is used to recognise the protein structure of interest. Such an unpredicted problem serves to emphasise the importance of understanding socially situated practice as the relationship between 'plans and situated actions' (Suchman 2006). The plan within this extended frame of practice is not disentangled from the material culture in which the situated actions occur, the plan is itself 'an act of embodiment' (Malafouris 2013, 236). As noted, the Western Blot protocol does not exist in isolation; the situated action is informed by previous embodied socio-material relations. The researcher comes to know the institutionally embedded affordances of the materials engaged with and these relations are skilfully negotiated as a means to achieve the intended function of the clear Western Blot image. Yet the researcher in this instance was unaware of a change in relations (the expiration of the antibody) that occurred in the intervening time between the initial experimentally valid Western Blot and the subsequent attempts to produce an image for publication. The socio-materially embedded plan assumed the material relations remained consistent, and thus the plan reflected the shifting institutional conditions, shifting from local experimental support, to the conceptual integration of internationally recognised publication standards of evidence presentation.

The Western Blot is valued differently by the researcher based upon the sociomaterial relations in which the blot was to be embedded. The same researcher engages with the material outcome of the collective co-operation of human and material actors in the same physical environment in distinctive ways. Such shifts in ways of thinking can be seen to emerge out of the relations the action has with the institutional conditions it serves to reproduce. In the first instance, the Western Blot serves to reproduce the ideal of scientific rigour, at the same time serving to reproduce the local cautious attitude to experimental practice that has developed over time. The Western Blot was used as a mechanism for checking that the product was inserted and cloned correctly. This is not a juxtaposition to the Western Blot produced for a scientific publication, rigour is certainly emphasised in the field of biomedical research publishing, the emphasis is shifted to produce a clear narrative in which noise and unexpected results that are 'known' to have led to this point are forgone for the polished, clean image. 
Latour and Woolgar (1979) describe the discrepancy between inscriptions of experimental practice and the results within academic publications as a process of deleting modalities, or mediating discourse associated with contingency and uncertainty within inscribed texts. Much as reported by Alač (2004), a large proportion of inscriptions that are produced in the laboratory are visual. At the core of Alač's analysis is the observation that the visual material produced is not a mode of representation, the fMRI images, reported in Alač's research, do not represent the brain. In the same vein the bands produced on the Western Blot do not represent the protein they measure. Instead, the Western Blot signifies the enactment of the material culture in which it is entangled. The distinctions between experimental and publication Western Blots has little to do with the information contained within them. Much in the same way described by Law and Mol (2008) with regard to Cumbrian Sheep, the distinction between the blots lies in the cognitive institution in which they are embedded and that which they serve to stabilize. However, furthering Law and Mol's claim I show that the very material structure of the Blot changes. Acting as a material sign, the blot substantiates the specific shared qualities of the presentation of a particular protein within the conceptual blend as an active participant in the stabilization process. The material substantiation that constitutes this process is akin to Myers' (2015) concept of rendering. Myers does not reduce the meaning of rendering to the embodied process by which modellers make observable otherwise imperceptible protein structures. Instead she constructs a polysemic meaning; rendering is also taken to signify the entangled coextensive process in which the amplification and animation of the protein structures amplifies and animates the practices of the modellers. The production of a 'clean' Western Blot recursively stabilizes the practices within which the researcher is situationally embedded. The 'clean' Western Blot signifies practices associated with scientific publishing; consequentially the situated discourse of the researcher is adapted. Following the production of the 'clean' Western Blot protocol as the transfer process is running, the researcher justifies the extra effort and use of resources used by emphasising the relationship of this experiment with the external research community. They note how they did not want to give the peer reviewer something to criticise in the submitted manuscript. This consideration, which spatially and temporally extends far beyond the boundaries of the laboratory, was absent in the initial experimental procedure of checking for the presence of the protein. To be clear, this is not to say that the structure of the 'clean' Western Blot changed the researcher's way of thinking; from an experimental to a publication logic, but it is to say that the Western Blot served to stabilize already enacted ways of thinking, rendering the later discussion by the researcher concerning academic publishing pressures situationally appropriate.

By adopting the radical approach to material agency put forward by Malafouris (2013) and others (Knappet and Malafouris 2008), which champions a nonanthropocentric approach to agency, this section has emphasised the crucial dependence of human and material agents in the production of the Western Blot. It is worth emphasising that the materials that furnish the experimental space of the lab bench are not endowed with agency by researcher. The researcher does not impose form on the Western Blot. Instead, it is from the tightly coupled, embodied relations between the researcher and the material culture that agency is enacted, and the institutional cognitive ecology is produced and stabilized. 
The Western Blot is helpful in sketching out the reflexive relationship actors have with the socio-material organisation of the worlds that they enact, or can enact. The above shows how very different ways of thinking are enacted by the same assemblage of brains, bodies and things relative to the overarching institutional social organisation. The cognitive institutions of checking for proteins and publication preparation, encapsulate past collective experiences and rules that guide what is expected in the unfolding of practice. The case study equally serves as an illustration of the metacognitive location of institutions, in that their existence crucially depends on disciplined sociomaterial relations to re-produce and sustain them, not on the physical space or the individual mind. Moreover, by examining the effect of changing socio-material dynamics, this section has shown, not only that some thought processes would not have been conceivable in isolation of the unfolding of tightly coupled socio-material relations; but also that as these relations shift, so does the externalisation of mental processes. Indeed, it does not make sense to consider the constituent mental processes in isolation of these conditions, as Malafouris (2013) succinctly notes, enactive thought 'is essentially constituted through an act of collaboration between human and material agency' (236). However, I have not yet discussed how actors become co-constitutively intertwined in relational practices. While material signs help to explicate the ongoing relations, material signs can be, at the same time embedded within plural relationalities, and the cultural forms stabilised by these anchoring devices are often only visible to those already entangled. This final empirical section will explore the hypothesis of the extended mind (Malafouris 2013) as the mode by which actors come to mutually constitute cognitive worlds.

\section{The extended mind}

The approach to the extended mind taken here is distinct from extended cognition (Clark and Chalmers 1998), in that the brain and the body are integral to the cognitive process, but not to the detriment of the value of the surrounding material culture. Material culture is not relegated to the role of participants in cognitive processes; it is instead positioned as actively constituting the mind. Material culture is not passive to the will of the human actor. As shown in the previous section, materials are active participants in the co-constitution of the cognitive network of situated action (Malafouris 2008, 2013). The materials shape the thought of the researcher as the researcher influences the actions of the materials engaged with in practice. That being said, one does not want to dismiss the place of the human brain and body, after all the body is the means by which we interact with the world and is coextensive with the way we think. It is the productive nature of this embodied relationship with an active material world that will be explored in this section with regard to the place of the lab book within the cognitive ecology of laboratory practices.

\subsection{The place of the lab book in (re)producing experimental protocols}

Every researcher in the laboratory uses A4 scale black covered lab books for each research project they are working on. One could draw upon theories of 'extended cognition' (Clark and Chalmers 1998; Clark 1997), to say that the researchers use the 
lab book to off load the cognitive burden of remembering previous experimental experiences, protocols and findings into a material container. Whilst of course, this is one way in which the book is engaged with, it could also be positioned as a platform for performing the social-materially entangled institutions to audiences not immediately present. This is certainly legitimate, and the researchers are conscious of this when deciding what to write down on scrap paper, such as rough equations, and what to commit to the permanent record of the lab. This understanding broadens the memory capacity of the lab book to a form of carefully curated institutional memory (EvansPritchard 1939; Douglas 1986), serving to reproduce the precise laboratory narrative contained there within. The lab book in this case is positioned as, what Goodwin (2017) terms a predecessor, serving to shape future practices in the absence of the researcher who produced the document. Such a document is unique in that it meticulously reconstructs the uncertainties, failures and non-linear process of experimental practice over time as a way to inform future practice and pre-empt inevitable problems. While a major official function of the lab book is to serve as a record of practice that could be reproduced based upon the inscribed text, much like the clinical notes reported on by Garfinkel (1967), the written record would seem incomplete outside of the organisational system in which it is instituted. Garfinkel (1967) refers to this as 'indexicality', or the indexical meaning. The text is tightly coupled to social organisational practices and serves as a material sign enabling actors to understand the unfolding of action around them. One such process observed in the laboratory originating from this contextually constrained, relational engagement between the researcher and the lab book emphasises the importance of understanding situated instances of material engagement as unfolding and enfolded within a broader cognitive ecology.

The researcher was working on a protocol to look for a particular mutated protein in an insoluble protein pellet. In most cases it is sufficient to simply analyse the soluble protein, so the protein pellet protocol is rarely used. The researcher selected the lab book that containing the experimental protocol and intuitively found the page that the protocol was on: 'I know it is in this book, I know it is near the back, and I know there is an image on the page'. The researcher recognised the image of the Western Blot produced by this experiment as distinct from the others contained within the book. They were then able to recall each stage of this protocol from a single page, which described an experimental protocol undertaken only one time three years ago. Moreover, the researcher is able to recall and circumvent specific issues that arose the first time the experiment was undertaken. They are able to optimize in-situ, they remember that the mixture can be quite viscous and is prone to sticking in the pipette tips, so larger tips are used. From a different experiment using similar materials, the researcher recalls that a particular magnet should be used for the magnetic mixer because of the dimensions of the flask needed to mix the protein mixture as to prevent it 'bouncing around'. Whilst this appears to be an incredible feat of memory, under further scrutiny it can be examined in relation to the extended experimental cognitive ecology of laboratory, instances are recalled and navigated where the researcher interacts with the materials around them.

Such an approach shifts the process from one of recalling the details of an experimental protocol, to a process of actively re-constructing the protocol. It is more akin to assembling the protocol afresh, made possible by the embodied enfolding of the 
researcher within the socio-material ecology of the experimental laboratory. Collectively the embodied engagement between the researcher, the lab book, the pipettes, the magnetic mixer, the centrifuge, the various sizes and shapes of flasks and tubes - all specifically organised around the experimental process of finding whether a protein is present or absent in a viscous mixture - enables the accurate reproduction of an experimental procedure, in a way that isolating the embodied interaction between the researcher and the lab book would not.

The above serves to emphasise the place of material culture in the structuring of thought processes. As Douglas and Isherwood (1979) note in reference to Lévi-Strauss (1962), materials (commodities) are 'good for thinking' (41). Malafouris takes this further in his claim that human thought is inseparably linked to the external, material world. We essentially think 'through and with matter' (Malafouris 2013, 236) across 'Hylonoetic fields', which are described as situated supra-organismic mind-scapes, constitutively intertwining human action, material culture and the social environment. It stands to reason that as the organisation of the matter that we think through and with changes within embodied practice, the way we think changes (Malafouris 2016c, 2018). This can be explored though the engagement with the protocol in the lab book for creating a buffer for the protein pellet analysis described earlier.

Much like the protein pellet protocol, the buffer requires very specific amounts of chemicals. However, unlike the protein pellet protocol the researcher is unable to recall the location of the chemical components and regularly revisits the written protocol. The researcher had to write down the name of the primary ingredient, sodium hydroxide, the compound formula that was noted on the bottle $(\mathrm{NaOH})$, as well as the specific quantity needed. For the protein pellet protocol, the researcher was able to recall the specific location and name of the constituent elements without any notes. Upon further enquiry the researcher commented that other people regularly use this chemical and it is not always put back in the same place. The constituent elements for the protein pellet protocol were created by the researcher and stored in their own designated freezer space; whereas the bulk chemicals are stored in a shared area of the laboratory. What this comparison serves to emphasise is that even specific cognitive processes, such as memory formation and recall are essentially and inseparably intertwined with the process of embodied material engagement, 'where the hand meets the stone' (Malafouris 2013, 236). There is the expectation that bulk chemical storage area will be disorganised, thus embodied experience dictates that it would not be much use to retain information regarding the specific location of a chemical.

The extended mind is explored as the embodied process of thinking with the material world. It is the mechanism by which the brain and body interacts and becomes mutually constitutive with the world around them. I have shown quite literally, how embodied practice with the plural material organisation of the laboratory affects thought processes traditionally confined within the brain of the individual.

\section{Conclusion}

This preceding holds that the world is not visible in the objects that furnish it, nor is it visible in isolated action or interaction; the world only becomes visible insofar as it is enactively produced and re-produced via distributed relations across the cognitive 
ecology of the laboratory. There are many worlds or institutional ecologies that actors dwell in, defined by distinct relational patterns of engagement. This is explored here as the process of cognitive shifts, or the way in which agency is enacted relative to the patterns of collective actions as they unfold. The impact of this relational understanding of agency for conceptions of the mind are encapsulated in Strathern (1996) account of Melanesian agency, in which she convincingly shows that the mind cannot be separated from the enactment of elaborate social relations and history, it cannot be contained in the individual person. A sentiment Barad (2003) captures concisely: "Agency is not an attribute but the ongoing reconfiguring of the world" (818). I position cognition as the enactment of complex and shifting social-material relations that serve to configure and re-configure the world. Under this paradigm agency is not an attribute but a consequence of enacted participation within the unfolding of relations in a social world.

Decoupling agency from the actor, human or material, offers theoretical support for the assertion that the form of thought engaged with by actors shifts based upon the enactment of social relations across institutional ecologies. The subtle distinction here is to diverge from saying that the enacted agency of the actor changes, to stating that the actor enacts a different form of institutionally reproduced agency. Agency becomes the possession of the relationally enacted world. The engagement within the collective socio-material relations that produce the world, via the enactment of a particular form of agency, is the mechanism by which an actor becomes co-ordinated with it.

The 3 constitutive hypotheses of MET have been employed here as the broad scaffold from which the situated negotiation of the dynamic, social material organisation of the biomedical research laboratory, by the researcher can be explored. Malafouris (2013) offers these hypotheses as a way to develop a more complete understanding of the extended and enactive constitution of the mind within material engagement. Drawing on some implicit assumptions of dynamicity and temporal contingency concerning agency within MET, this paper explores how and when agency is constituted and re-constituted within the biomedical research laboratory. Exploring the day-to-day experimental processes of the biomedical research laboratory through the lens of MET has provided empirical support for the dynamic, situationally contingent conception of cognition put forward in this paper. I have shown how the changing social and material organisation of the laboratory has dramatic consequences on the way that researchers think and engage with the materials around them; from the effect of particular material ecologies on memory formation and recall, to the productive capacity of socially and materially constituted metacognitive institutions within experimental practices, as shown in the multiple framings of the production of the Western Blot.

Perhaps more importantly, by exploring the situated cognitive flexibility of researchers within situated practice, the coextensive relationship between the brain, body and material organisation of the laboratory is substantiated within and across shifting complexes of socio-material relations. Whilst Hutchins and Alač (2004) have previously emphasised the importance of the material organisation of the laboratory on the mutual constitution of the active process of cognition, there has been little consideration of the consequences of the dynamic socio-material organisation of the laboratory on the relational enactment of cognition in practice. Such considerations far extend the cognitive demands put upon the research scientist as they negotiate the complex and dynamic cognitive organisation of biomedical laboratory practice. 
Open Access This article is distributed under the terms of the Creative Commons Attribution 4.0 International License (http://creativecommons.org/licenses/by/4.0/), which permits unrestricted use, distribution, and reproduction in any medium, provided you give appropriate credit to the original author(s) and the source, provide a link to the Creative Commons license, and indicate if changes were made.

\section{References}

Adam, A. (1998). Artificial knowing: Gender and the thinking machine. New York: Routledge.

Alač, M. (2004). Negotiating pictures of numbers. Social Epistemology, 18(2-3), 199-214.

Barad, K. (2003). Posthumanist performativity: Toward an understanding of how matter comes to matter. Signs, 28(3), 801-831.

Bartlett, A., Penders, B., \& Lewis, J. (2017). Bioinformatics: Indispensable, yet hidden in plain sight? BMC Bioinformatics, $18(1), 311$.

Clark, A. (1997). Being there: Putting the brain, body and world together again. Cambridge: MIT Press.

Clark, A., \& Chalmers, D. (1998). The extended mind. Analysis, 58(1), 7-19.

Di Paolo, E. (2009). Editorial: The social and enactive mind. Phenomenology and the Cognitive Sciences., $8(4), 409-415$.

Douglas, M. (1986). How Institutions Think. New York: Syracuse University Press.

Douglas, M., \& Isherwood, B. (1979). The world of goods: Towards an anthropology of consumption. New York: Basic Books.

Evans-Pritchard, E. E. (1939). Nuer Time Reckoning. Africa, 12(2), 186-216.

Fauconnier, G., \& Turner, M. (2002). The way we think. New York: Basic Books.

Gallagher, S. (2013). The socially extended mind. Cognitive Systems Research, 25-26, 4-12.

Gallagher, S. (2017). Enactivist interventions, rethinking the mind. Oxford University Press.

Garfinkel, H. (1967). Studies in ethnomethodology. New Jersey: Prentice Hall.

Gibson, J. (1977). The theory of affordances. In R. Shaw \& J. Branshaw (Eds.), Perceiving, Acting and Knowing (pp. 67-82). New Jersey: Erlbaum.

Goffman, E. (1956). The presentation of self in everyday life. New York: Random House.

Goodwin, C. (2017). Co-operative action. Cambridge: Cambridge University Press.

Gosden, C. (2008). Social Ontologies. Philosophical Transactions of the Royal Society B: Biological Sciences, 363(1499), 2003-2010.

Heidegger, M. (1927)[1962]. Being and Time. Trans: J. Macquarrie and E. Robinson. Oxford: Blackwell Publishers.

Hollan, J., Hutchins, E., \& Kirsh, D. (2000). Distributed cognition: Toward a new Foundation for humancomputer interaction research. ACM Transactions on Computer-Human Interaction, 7(2), 174-196.

Hutchins, E. (1995a). How a cockpit remembers its speeds. Cognitive Sciences., 19, 265-288.

Hutchins, E. (1995b). Cognition in The Wild. Cambridge: MIT Press.

Hutchins, E., Alač, M. (2004). I see what you are saying: Action as cognition in fMRI brain mapping practice. Journal of Cognition and Culture, 4(3), 629-661.

Hutchins, E. (2005). Material anchors for conceptual blends. Journal of Pragmatics, 37(10), 1555-1577.

Hutchins, E. (2010). Cognitive Ecology. Topics in Cognitive Science, 2(4), 701-715.

Hutto, D., \& Myin, E. (2013). Radicalizing enactivism: basic minds without content. Cambridge: MIT Press.

Iliopoulos, A. (2016). The evolution of material signification: Tracing the origins of symbolic body ornamentation through a pragmatic and enactive theory of cognitive semiotics. Signs and Society, 4(2), 244-277.

Keating, P., \& Cambrosio, A. (2003). Biomedical Platforms: Realigning the Normal and Pathological in LateTwentieth-Century Medicine. Cambridge: MIT Press.

Knappett, C., \& Malafouris, L. (2008). Material agency: Towards a non-anthropocentric approach. New York: Springer.

Latour, B., \& Woolgar, S. (1979). Laboratory life: The construction of scientific facts. Princeton: Princeton University Press.

Law, J., \& Mol, A. (2008). The actor-enacted: Cumbrian sheep in 2001. In C. Knappett \& L. Malafouris (Eds.), Material agency: Towards a non-anthropocentric approach (pp. 57-78). New York: Springer.

Lévi-Strauss, C. (1962). Totemism. London: Merlin Books.

Lock, M., \& Nguyen, V. K. (2018). An anthropology of biomedicine. Oxford: Blackwell.

Lynch, M. (1991). Laboratory space and the technological complex: An investigation of topical contextures. Science in Context, 4(1), 51-78. 
Malafouris, L. (2004). The cognitive basis of material engagement: Where brain, body and culture conflate. In E. DeMarrais, C. Gosden, \& C. Renfrew (Eds.), Rethinking materiality: The engagement of mind with the material world (pp. 53-62). Cambridge: McDonald Institute for Archaeological Research.

Malafouris, L. (2008). Between brains, bodies and things: Tectonoetic awareness and the extended self. Philosophical Transactions of the Royal Society of London, Series B, 363, 1993-2002.

Malafouris, L. (2009). "Neuroarchaeology": Exploring the links between neural and cultural plasticity. Progress in Brain Research, 178, 253-261.

Malafouris, L. (2010). Metaplasticity and the human becoming: Principles of neuroarchaeology. Journal of Anthropological Sciences, 88, 49-72.

Malafouris, L. (2013). How Things Shape the Mind: A Theory of Material Engagement. Cambridge: MIT Press.

Malafouris, L. (2015). Metaplasticity and the primacy of material engagement. Time and Mind, 8(4), 351-371.

Malafouris, L. (2016a). On human becoming and incompleteness: a material engagement approach to the study of embodiment in evolution and culture. In G. Etzelmüller \& C. Tewes (Eds.), Embodiment in evolution and culture (pp. 289-305). Tübingen: Mohr Siebeck.

Malafouris, L. (2016b). Material engagement and the embodied mind. In T. Wynn \& F. L. Coolidge (Eds.), Cognitive models in Palaeolithic archaeology (pp. 69-82). Oxford: Oxford University Press.

Malafouris, L. (2016c). Hylonoetics: on the priority of material engagement. In K. Grigoriadis (Eds.), Mixed matters: A multi-material design compendium (pp. 140-146). Berlin: Jovis Verlag.

Malafouris, L. (2018). Bringing things to mind: 4Es and Material Engagement. In A. Newen, L. de Bruin, \& S. Gallagher (Eds.), The Oxford Handbook of 4E Cognition (pp. 755-71). Oxford: Oxford University Press.

Maturana, H., \& Varela, F. (1980). Autopoiesis and cognition: The realization of the living. In Boston Studies in the Philosophy of Science 42. Dordrecht: D. Reidel Publishing Company.

Merleau-Ponty, M. (1962). Phenomenology of Perception. Abingdon. Routledge.

Mol, A. (2002). The Body Multiple. Durham: Duke University Press.

Myers, N. (2015). Rendering Life Molecular: Models, Modellers and Excitable Matter. Duhram: Duke University Press.

Newen, A., De Bruin, L., \& Gallagher, S. (Eds.). (2018). The Oxford handbook of $4 E$ cognition. Oxford University Press.

Oudshoorn, N. (2015). Sustaining cyborgs: Sensing and tuning agencies of pacemakers and implantable cardioverter defibrillators. Social Studies of Science, 45(1), 56-76.

Stewart, J., Gapenne, O., \& Di Paolo, E. (2011). Enaction: Toward a new paradigm for cognitive science. Cambridge: MIT Press.

Strathern, M. (1996). Cutting the network. Journal of the Royal Anthropological Institute, 2(3), 517-535.

Suchman, L. (2006). Human-machine reconfigurations: Plans and situated actions. Cambridge: Cambridge University Press.

Thompson, E., \& Stapleton, M. (2009). Making sense of sense-making: Reflections on enactive and extended mind theories. Topoi, 28(1), 23-30.

Valera, F., Thompson, E., \& Rosche, E. (1991). The embodied mind: Cognitive science and human experience. Cambridge: MIT Press. 